Pacific Journal of Mathematics

SOME STRUCTURE THEOREMS FOR A CLASS OF BANACH 


\title{
SOME STRUCTURE THEOREMS FOR A CLASS \\ OF BANACH ALGEBRAS
}

\author{
ALFRED B. WILLCOX
}

o. Introduction. The purpose of this paper is the presentation of a structure theory for a class of Banach algebras which we define below and call GS-algebras. This class includes the commutative regular $B$-algebras of Silov [9] and many of our results generalize theorems and techniques of that author. In addition, several interesting types of non-commutative $B$-algebras (listed in $\S 1$ ) which have been studied previously only individually and from rather widely deffering points of view are included in the class of $G S$-algebras. In $\S 1$ we introduce some basic definitions and prove several fundamental theorems. $\S 2$ contains some theorems on the structure of closed two-sided ideals in certain $G S$-algebras, and in $\S 3$ we present a decomposition theory for such_algebras.

1. Preliminary definitions and results. It is assumed that the reader is familiar to a certain extent with the theory of rings and ideals and the basic theory of $B$-algebras. The theory of regular commutative $B$ algebras can be found in [5], [6], or [9]. In this paper ideal will mean two-sided ideal. Consider a $B$-algebra $R$ with structure space $S(R)$. $S(R)$ is the collection of maximal regular ideals of $R$ with the standard Stone-Jacobson topology which is defined as follows: the closure $F^{c}$ of a set $F \subset S(R)$ is $\left\{M \in S(R) \mid M \supset \cap M^{\prime}, M^{\prime} \in F\right\}$. The terminology is rendered somewhat more manageable by defining the kernel $k(F)$ of a set $F$ in $S(R)$ to be the intersection of all maximal regular ideals in $F$ and the hull $h(I)$ of an ideal $I$ in $R$ to be the set of all elements of $S(R)$ which contain $I$. Then the hulls are the closed sets in $S(R)$. If $F=h(I)$ we say that $I$ belongs to $F . S(R)$ is, in general, a $T_{1}$-space and it is compact if $R$ contains an identity. We say that $R$ is stronglysemi-simple (s.s.s.) if the intersection of all maximal regular ideals is zero.

If $R$ has an identity then the theory we present below can be car-

Received September 2, 1954; presented in slightly different form to the American Mathematical Society, February 28, 1953. This paper is essentially a dissertation presented to the faculty of Yale University in partial fulfillment of the requirements for the degree of Doctor of Philosophy and was prepared while the author was an Atomic Energy Commission predoctoral fellow. The author wishes to express his thanks to Professor C. E. Rickart for his help and encouragement during the time when this paper was in preparation, and to the referees whose suggestions have led to considerable improvements in some proofs. 
ried through under the single basic condition

(A) $S(R)$ is Hausdorff.

However, in case $R$ has no identity further conditions are necessary, and, while individual theorems can be proved under various weaker conditions, one needs for any substantial part of the theory properties at least as strong as condition

(B) every point in $S(R)$ is contained in an open set whose closure has regular kernel. (An ideal $I$ is regular if $R / I$ has an identity.)

A $B$-algebra which satisfies Conditions (A) and (B) will be called a GS-algebra.

The following is a device which will be useful in subsequent proofs. Let $S^{\prime}(R)$ be the space formed by adding to $S(R)$ a point $\infty$ and defining a neighborhood of $\infty$ to be $\infty$ together with the complement in $S(R)$ of a hull whose kernel is regular.

THEOREM. $S^{\prime}(R)$ is a compact $T_{1}$-space homeomorphic to the structure space of the algebra $R^{\prime}$ formed by adjoining a unit to $R$. If $R$ is a GS-algebra then $S^{\prime}(R)$ is Hausdorff, and conversely. In this case $S^{\prime}(R)$ is the ordinary one-point compactification of $S(R)$.

Proof. The proof of the first statement is contained in [8, Th. 2.8] and we will omit it here. It is evident that Conditions (A) and (B) are equivalent to the Hausdorff conditions in $S^{\prime}(R)$. The last statement follows from the fact that the intersection of two neighborhoods of $\infty$ is a neighborhood of $\infty$ which is equivalent to the assertion that if $F_{1}$ and $F_{2}$ are hulls with regular kernels then $k\left(F_{1} \cup F_{2}\right)$ is regular. This, in turn, is equivalent to the assertion that the intersection of two regular ideals, $I_{1}$ and $I_{2}$, is regular, which follows from the observation that if $e_{i}$ is a unit modulo $I_{i}$ then $e_{1}+e_{2}-e_{2} e_{1}$ is a unit modulo $I_{1} \cap I_{2}$. Knowing this one verifies immediately that in a $G S$-algebra the kernel of a compact set in $S(R)$ is regular from which the last statement of the theorem follows.

While we will avoid in statements of theorems the somewhat artificial device of adjoining a unit to $R$, it will help us to recall the above facts in later proofs.

For $x \in R$ and $M \in S(R)$ we denote by $x(M)$ the image of $x$ in the difference algebra $R / M$ and by $\|x(M)\|$ the norm of this element of $R / M$. We shall make extensive use of the functions $x(M)$ for fixed $x \in R$ defined on the space $S(R)$ and having values in the algebras $R / M$, and of the real valued functions $\|x(M)\|$. 
Definition 1.1. The GS-algebra $R$ satisfies condition $(P)$ if for any compact subset $K$ of $S(R)$ covered by open sets $V_{1}, \cdots, V_{n}$ there exist elements $y_{1}, \cdots, y_{n}$ of $R$ such that $(1) \sum y_{i}(M)=1, M \in K$, and (2) $y_{i}(M)$ $=0$ outside $V_{i}$.

Lemma 1.1.1. (a) Any proper regular ideal in $R$ is contained in a maximal regular ideal, that is, $h(I) \neq \phi$.

(b) If $I$ is a closed ideal in $R$ then $M \rightarrow M / I$ is a homeomorphism of $h(I)$ onto $S(R / I)$. (See [2] or [8].)

THEOREM 1.1. If $I_{1}, \cdots, I_{n}$ are ideals in $R$ with at least one $I_{i}$ regular and $\bigcap_{i} h\left(I_{i}\right)=\phi$ then $I_{1}+\cdots+I_{n}=R$.

Proof. $h\left(I_{1}+\cdots+I_{n}\right)=\bigcap_{i} h\left(I_{i}\right)=\phi$ and $I_{1}+\cdots+I_{n}$ is a regular ideal. Lemma 1.1.1(b) completes the proof.

Corollary 1. 1. 1. Let $I$ be an ideal in $R, F=h(I)$, and $F_{0}$ be a hull with regular kernel and disjoint from $F$. Then there exists an element $x \in I$ such that $x(M)=1$ for all $M \in F_{0}$.

Proof. $I+k\left(F_{0}\right)=R$ by Theorem 1.1 so there exist $x_{1}, x_{2}$ in $I$ and $k\left(F_{0}\right)$ respectively such that $x_{1}+x_{2}$ is a unit modulo each $M \in F_{0}$. The conclusion follows immediately.

By the above remarks, if $R$ is a $G S$-algebra then the set $F_{0}$ in Corollary 1.1 .1 can be any compact hull disjoint from $F$.

COROLLARY 1.1.2. If $R$ is a GS-algebra then $R$ satisfies condition $(P)$.

Proof. Let $K$ be compact and covered by $V_{1}, \cdots, V_{n}$. Since $S^{\prime}(R)$ is normal we can obtain a refinement $U_{1}, \cdots, U_{n}$ of $V_{1}, \cdots, V_{n}$ covering $K$ and such that $U_{i}^{c} \subset V_{i}$. By Condition (B) we can assume that $U_{i}^{c}$ is compact. Let $C_{i}$ be the complement of $U_{i}$, then $C_{i}$ is a hull. If $I_{i}=k\left(C_{i}\right)$ then $\bigcap_{i} h\left(I_{i}\right)=\bigcap_{i} C_{i}=\mathscr{C}\left(\bigcup_{i} U_{i}\right) \subset \mathscr{C}(K)$. Thus $h\left(I_{1}+\cdots\right.$ $\left.+I_{n}\right) \cap K=\phi$ so there exist $x_{i} \in I_{i}$ such that $\left(x_{1}+\cdots+x_{n}\right)(M)=1$ on $K$. But $x_{i}(M)=0$ for $M \in k\left(I_{i}\right)$, that is, for $M$ outside $U_{i}$. But $U_{i} \subset V_{i}$.

Definition 1.2. An ideal $I$ is primary if it is contained in a unique maximal regular ideal. A $B$-algebra is primary if $S(R)$ consists 
of a single point. (By Lemma 1.1.1 $I$ is primary if and only if $R / I$ is a primary algebra.)

We proceed to show the existence of certain special primary ideals in GS-algebras.

Definition 1.3. Let $F$ be a hull in $S(R)$. We define $J(F)$ to be the set of all $x \in R$ such that $x(M)=0$ in some open set containing $F$. $J(\infty)$ is the set of all $x$ such that $x(M)=0$ outside a compact set. $\Im(F)=J(F) \cap J(\infty)$.

TheOREM 1.2. Let $R$ be a s.s.s. GS-algebra and $F$ be a hull in $S(R)$. Then

(a) $h(J(\infty))=\phi$,

(b) $h(\Im(F))=h(J(F))=F$, and

(c) if $h(I)=F$ then $I \supset \Im(F)$.

Thus the closure $\Im^{c}(F)$ of $\Im(F)$ is the smallest closed ideal belonging to $F$.

Proof. (a) is a simple consequence of Corollary 1.1.1. To establish (b) it is sufficient to show that $F \supset h(J(F))$ since the opposite inclusion and the first equality of (b) are both obvious. If $M_{0} \supset J(F)$ then let $G$ be an open set containing $F$, and pick $x \in k(G)$. Then $x$ is in $J(F)$ by definition so $x$ is in $M_{0}$. Thus $M_{0}$ is in the closure of an arbitrary open set containing $F . S(R)$, being a subspace of the topologically regular space $S^{\prime}(R)$, is topologically regular, so it follows that $M_{0}$ is in $F$. Thus $F \supset h(J(F))$. We verify (c) as follows. Let $h(I)=F$ and let $y$ be in $\Im(F)$. Then there exist an open set $U$ containing $F$ and a compact hull $K$ such that $y(M)=0, \quad M \in U \cup \mathscr{C}(K)$. Let $F_{1}=\mathscr{C}(U) \cap$ $K$, a compact hull disjoint from $F$. By Corollary 1.1.1. there exists $x \in I$ such that $x(M)=1$ for $M \in F_{1}$ and it follows that $y(M)=x(M) y(M)$ on $S(R)$. By semi-simplicity, then, $y=x y$ so $y$ is in $I$. Thus $\Im(F) \subset I$.

If $F$ is the hull in $S(R)$ consisting of the single point $M$ we denote $\Im(F)$ by $\Im(M)$. Thus in a s.s.s. GS-algebra every maximal regular ideal $M$ contains a minimal closed primary ideal $\Im^{c}(M)$.

We conclude this section with some examples of GS-algebras.

1. As we have observed above, any commutative regular $B$-algebra 
is a $G S$-algebra. Examples of these can be found in [5], [6], or [9]. An important example is the group algebra ( $L^{1}$-algebra) of a locally compact abelian group.

2. Any c.c.-algebra (right and left multiplications are completely continuous operators) has discrete structure space [3]. It follows immediately that the algebra is a GS-algebra. An example is the group algebra of a compact group.

3. The group algebra of the direct product of a locally compact abelian group and a compact group is also a GS-algebra. It is our intention to include a proof of this fact in a future note.

4. Certain $C^{*}$-algebras studied by Kaplansky (see [3] and [4]) are $G S$-algebras. In particular, any $C^{*}$-algebra with identity for which the functions $\|x(M)\|$ are continuous satisfies Condition (A) and is therefore a GS-algebra. Also, any weakly central $C^{*}$-algebra (see Definition 1.4 below) is a GS-algebra as we note in Theorem 1.3. Examples of such algebras can be found in [3].

5. Misonou [7] shows that any $W^{*}$-algebra is weakly central (hence a $G S$-algebra) and Wright has proved [10] that the same is true for any $A W^{*}$-algebra.

6. The $B$-algebra $D_{n}^{\prime}$ of all $n$ by $n$ matrices with entries which are continuously differentiable complex functions on $[0,1]$ is a $G S$ algebra. The norm is $\|x\|=\sup |x(t)|+\sup \left|x^{\prime}(t)\right|$ where $|x(t)|=$ $\sum_{i, j}\left|x_{i, j}(t)\right|$ and $\left|x^{\prime}(t)\right|=\sum_{i, j}\left|x_{i, j}^{\prime}(t)\right|$. A maximal regular ideal $M_{t_{0}}$ is the set of all $x \in D_{n}^{\prime}$ such that $x_{i, j}\left(t_{0}\right)=0$, all $i, j$ and some $t_{0}$ in the interval. $S\left(D_{n}^{\prime}\right)$ is homeomorphic with the interval under the mapping $t \rightarrow M_{t}$. This is an algebra in which primary ideals arise quite naturally. The ideal $J^{c}\left(t_{0}\right)$ consists of all $x$ such that $x_{i, j}\left(t_{0}\right)=x_{i, j}^{\prime}\left(t_{0}\right)=0$.

Definition 1.4. The $B$-algebra $R$ with center $Z$ is weakly central if maximal regular ideals of $R$ intersect $Z$ in distinct proper ideals of $Z$, that is, if (1) $M \cap Z \neq Z$ for all $M \in S(R)$ and (2) $M_{1} \neq M_{2}$ implies that $M_{1} \cap Z \neq M_{2} \cap Z$.

The following is a generalization to our setting of a theorem due to Kaplansky [3, Th. 9.1]. We omit the proof since Kaplansky's proof suffices with comparatively few changes. 
Theorem 1.3. Let $R$ be a B-algebra with regular center $Z$. Then the mapping $M \rightarrow M \cap Z$ is a continuous mapping of $S(R)$ onto $T$, a closed subset of $S(Z)$. If $R$ is s.s.s. then $T=S(Z)$. If $R$ is also weakly central then $S(R)$ is homeomorphic to $S(Z)$ and $R$ is a GS-algebra.

2. Ideal Structure. Frequently much can be learned about the structure of an algebra if information about the structure of its ideals is available. The type of information which is most commonly sought is that which says that an ideal is the intersection or direct sum of more special ideals (for example, primary or maximal regular ideals). Concentrating on intersection as the method of decomposition we prove in this section some results of this nature. The following questions naturally arise:

(a) Is every ideal (not necessarily regular) contained in a maximal regular ideal?

(b) When can a closed ideal be written as an intersection of maximal regular ideals or an intersection of closed primary ideals?

(c) When is a closed primary ideal necessarily maximal regular?

Some of the theorems in this section are generalizations of theorems due to Silov which can be found for the commutative case in [5]. Theorems 2.7 and 2.8 were suggested to the author by Kaplansky's use in [4] of partitions.

If $R$ is a $G S$-algebra, Theorem 1.2 applied to the case where $F$ is the vacuous set shows that the answer to (a) is in the affirmative if $J(\infty)=R$. In this case we shall call $R$ Tauberian. The statements of many subsequent theorems are somewhat simplified in a Tauberian GSalgebra. The algebras in examples (1), (2), and (3) in $\S 1$ are Tauberian.

Definition 2.1. The $B$-algebra $R$ is called an $N$-algebra if every closed ideal with non-vacuous hull is an intersection of maximal regular ideals. $R$ is called an $N^{*}$-algebra if every closed primary ideal is maximal.

It is clear that an $N$-algebra is an $N^{*}$-algebra. The converse is not true in general, but it is the purpose of some of the theorems below to exhibit circumstances under which it is. From the definition and Theorem 1.2 we have the following criteria. For $x \in R$ define $h(x)$ to be $\{M \mid x \in M\}$.

THEOREM 2.1. The s.s.s. GS-algebra $R$ is an N-algebra $\left(N^{*}\right.$-algebra) 
if and only if for every $x \in R$ (and every $M_{0} \in h(x)$ ) there exists a sequence $\left\{x_{n}\right\}$ in $R$ such that $x_{n}(M)=x(M)$ on a neighborhood $G_{n}$ of $h(x)$ (of $M_{0}$ ) and outside a compact set $K_{n}$, and such that $\left\|x_{n}\right\| \rightarrow 0$.

Proof. Simply note that by Theorem 1.2 an ideal $I$ in $R$ is the kernel of its hull if and only if for every $x \in I$ there exists a sequence $\left\{x_{n}\right\}$ in $R$ such that $x_{n}(M)=x(M)$ on a neighborhood $G_{n}$ of $h(I)$ and outside a compact set $K_{n}$, and such that $\left\|x_{n}\right\| \rightarrow 0$.

Definition 2.2. Let $f$ be a function defined on $S(R)$ such that $f(M)$ is an element of $R / M$ for each $M \in S(R)$. $f$ belongs locally to the ideal $I$ (which may be all of $R$ ) at the point $M_{0} \in S(R)($ at $\infty)$ if there exists a neighborhood $U$ of $M_{0}$ (of $\infty$ ) and an $x \in I$ such that $f(M)=$ $x(M)$ on $U . f$ belongs locally to $I$ if it belongs locally to $I$ at every point of $S(R)$ and at $\infty$.

Theorem 2.2. Let $R$ be a s.s.s. GS-algebra with $x \in R$. If $x(M)$ belongs locally to the ideal $I$ at every point in $h(I)$ and at $\infty$ then $x$ is in $I$.

Proof. We note first that since $S(R)$ is topologically regular $x$ belongs locally to $I$ at any point in $S(R)-h(I)$. Let $M_{0}$ be such a point. Then there exists a neighborhood $U$ of $M_{0}$ whose closure is compact and does not intersect $h(I)$. By Corollary 1.1 .1 we can find $u \in I$ such that $u(M)=1$ on $U$. $\quad x u$ is in $I$ and $x(M)=(x u)(M)$ on $U$. Thus, under the assumptions of the theorem, $x$ belongs locally to $I$.

There exists $y_{\infty} \in I$ such that $y_{\infty}(M)=x(M)$ outside a compact hull $K$. By compactness and assumption, $K$ can be covered by $n$ open sets $V_{1}, \cdots, V_{n}$ and $y_{1}, \cdots, y_{n} \in I$ can be chosen so that $y_{i}(M)=x(M)$ on $V_{i}$. By Corollary 1.1.2 there exist $x_{1}, \cdots, x_{n}$ such that $\sum x_{i}(M)=1$ on $K$ and $x_{i}(M)=0$ outside $V_{i}$. Now $x_{i} y_{i}(M)=x_{i}(M) x(M)$ on $\cup_{i} V_{i}$. Thus $\sum x_{i} y_{i}(M)=x(M)$ on $\bigcup_{i} V_{i}$. Let $y=\sum x_{i} y_{i}$. Another application of Corollary 1.1.1 yields an element $h$ of $R$ such that $h(M)=1$ on $F$, $h(M)=0$ on $\mathscr{C}\left(\cup V_{i}\right)$. Let $z=h\left(y-y_{\infty}\right)+y_{\infty} . \quad z$ is in $I$ since both $y$ and $y_{\infty}$ are, and it can easily be seen that $z(M)=x(M)$ for all $M \in S(R)$. This implies, by semi-simplicity, that $x=z \in I$.

COROLLARY 2.2.1. In a s.s.s. GS-algebra any ideal with nonvacuous hull is an intersection of primary ideals. 
This corollary follows immediately from the theorem and its proof is left to the reader. The primary ideals are the ideals $I+\Im(M)$ for $M \in h(I)$. By Theorem 1.2, $I+\Im(M)$ is the smallest primary ideal containing $I$ and contained in $M$.

The following theorem is a small step in the direction of expressing a closed ideal as an intersection of closed primary ideals.

Theorem 2.3. Let $I$ be a closed ideal in the s.s.s. GS-algebra $R$. If $h(I)$ can be written as the union of two disjoint hulls $F_{1}, F_{2}$ at least one of which is compact then $I$ is the intersection of closed ideals $I_{1}, I_{2}$ with $h\left(I_{i}\right)=F_{i}$.

Proof. Let $I_{i}=\left[\Im\left(F_{i}\right), I\right]$ be the closed ideals generated by the ideals in the brackets. Clearly all that needs to be verified is that $I_{1} \cap$ $I, \subset I$.

Let $x$ be in $I_{1} \cap I_{2}$. Then $x=\lim \left(y_{n}^{(1)}+z_{n}^{(1)}\right)$ and also $x=\lim \left(y_{n}^{(2)}+z_{n}^{(2)}\right)$ where $y_{n}^{(i)}$ is in $I$ and $z_{n}^{(i)}$ is in $\Im\left(F_{i}\right)$. Suppose that $F_{1}$ is compact. Since $S^{\prime}(R)$ is normal there exist open sets $G_{1}$ and $G_{2}$ such that $G_{i} \supset F_{i}$ and $G_{1}^{c} \cap G_{2}^{c}=\phi$. We may assume that $G_{1}^{c}$ is compact. Thus by Corollary 1.1.1 there exists $h \in R$ such that $h(M)=1$ on $G_{1}$ and $h(M)=0$ on $G_{2}$. Then $x=x h+(x-x h)=\lim \left[y_{n}^{(1)} h+z_{n}^{(1)} h+\left(y_{n}^{(2)}-y_{n}^{(2)} h\right)+\left(z_{n}^{(2)}-z_{n}^{(2)} h\right)\right]$. The first and third terms are in $I$ since the $y_{n}^{(i)}$ are in $I$ and it can easily be seen that the remaining terms vanish at $\infty$ and in a neighborhood of $F_{1} \cup F_{2}$ $=h(I)$. By Theorem 2.2, then, $x$ is a limit of elements of $I$ and hence is in $I$ itself.

Corollary 2.3.1. Let $R$ be a s.s.s. GS-algebra and $I$ be a closed ideal in $R$. If $M_{0}$ is an isolated point of $h(I)$ then $x \in\left[\Im\left(M_{0}\right), I\right]$ implies that $x$ belongs locxlly to $I$ at $M_{0}$. If $h(I)$ is compact then $x$ also belongs locally to $I$ at $\infty$.

COROLLARY 2.3.2. Under the above hypotheses, if $h(I)$ is discrete then $x \in I^{*}=\cap[\Im(M), I], M \in h(I)$, implies that $x$ belongs locally to $I$ at all points of $S(R)$. If $h(I)$ is compact then $I^{*}=1$, that is, $I$ is an intersection of closed primary ideals.

THEOREM 2.4. Let $R$ be a s.s.s. GS-algebra and $I$ be a closed ideal in $R$. Let $h^{(k)}(I)$ be the kth derived set of $h(I)$. Then if $x_{1}, \cdots, x_{m}$ $(m \geqq k+1)$ are all in $I^{*}=\bigcap[\Im(M), I], M \in h(I)$, their product belongs locally to $I$ at every isolated point of the set $h^{(k)}(I)$. If $h^{(k)}(I)$ is compact 
then the product also belongs locally to $I$ at $\infty$.

Proof. If $k=0$ this reduces to Corollary 2.3.1. We perform an induction on $k$. Suppose the statement is true for all integers up to and including $k$. Let $M_{0}$ be an isolated point of the set $h^{(k+1)}(I)$ and let $U\left(M_{0}\right)$ be a neighborhood of $M_{v}$ with compact closure not intersecting $h^{(k+1)}(I)-M_{0}$. Suppose $x_{1}, \cdots, x_{m}$ are all in $I^{*}$ with $m \geq k+2$. We can find another neighborhood $V_{1}$ of $M_{0}$ such that $V_{1} \subset V_{1}^{c} \subset U$. Let $h \in R$ be chosen so that $h(M)=1$ for $M \in V_{1}^{c}$ and $h(M)=0$ outside $U$. By assumption $x_{1}$ is in $\left[\Im\left(M_{0}\right), I\right]$ so $x_{1}=\lim \left(y_{n}+z_{n}\right), y_{n} \in I, z_{n} \in \Im\left(M_{0}\right)$. Thus $x_{1} x_{2} \cdots x_{m} h=\lim \left(y_{n} x_{2} \cdots x_{m} h+z_{n} x_{2} \cdots x_{m} h\right)$. The first term is obviously in $I$ and it is easy to see that the second term belongs locally to $I$ on $S(R)$ and at $\infty$. Hence $x_{1} x_{2} \cdots x_{m} h$ is in $I$ and, since $h(M)=1$ on a neighborhood of $M_{0}, x_{1} x_{2} \cdots x_{m}$ belongs locally to $I$ at $M_{0}$. The induction at $\infty$ is similar to the above, the important point being that if $h^{(k)}(I)$ is compact then $\infty$ is an isolated point of $h^{(k)}(I) \cup\{\infty\}$.

Corollary 2.4.1. Let $R$ be a s.s.s. GS-algcbra and $I$ be a closed ideal in $R$ such that $h^{(k)}(I)$ is void and $h^{(k-1)}(I)$ is compact (that is, $h^{(k-1)}(I) \cup\{\infty\}$ is discrete). Then, if $\left(I^{*}\right)^{k}$ is the ideal generated by products of $k$ elements of $I^{*},\left(I^{*}\right)^{k} \subset I$.

Next we turn our attention to the question of when a given $N^{*}$ algebra is an $N$-algebra. First we indicate briefly how Ditkin's theorem and Condition (D) [9] for commutative $B$-algebras generalize to our case.

Definition 2.3. The s.s.s. GS-algebra $R$ satisfies Condition (D) if for any $M_{0} \in S^{\prime}(R)\left(M_{0}\right.$ may be $\left.\infty\right)$ and $x \in \mathcal{S}^{\prime}\left(M_{0}\right)$ we have $x \in[I(x) \subset$ $\left.\mathfrak{J}\left(M_{0}\right)\right]^{\prime}$ where $I(x)$ is the principal two-sided ideal generated by $x$.

Theorem 2.5. Any s.s.s. N-algebra satisfies Condition (D).

Proof. If $x$ is in $\Im^{c}\left(M_{0}\right)$ then by Theorem 2.1 we can find $\left\{y_{n}\right\}$ such that $y_{n} \rightarrow x$ and $y_{n}$ is in $\Im(h(x))$. But $I(x)$ belongs to the hull $h(x)$ so $\Im(h(x)) \subset I(x)$. Thus $y_{n} \in I(x)$. An almost identical argument takes care of the case $M_{0}=\infty$.

For $x \in R$ and $I$ a closed ideal we denote by $P(x, I)$ the subset of $S(R)$ consisting of all $M$ at which $x$ docs not belong locally to $I$. 
Theorem 2.6. Let $R$ be a s.s.s. GS-algebra satisfying Condition (D). Let $x$ be in $R$ and $I$ be a closed ideal in $R$. Then any element $M_{0}$ in $P(x, I)$ for which $\mathfrak{\Im}^{c}\left(M_{0}\right)$ contains $x$ is a pcint of accumulation of $P(x, I)$.

Pronf. Suppose that $x$ is in $\Im^{c}\left(M_{0}\right)$ and $M_{0}$ is isolated in $P(x, I)$. Choose $U$ and $V$ open in $S(R)$ such that $M_{0} \in V \subset V^{c} \subset U, U^{c}$ compact and $U^{c} \cap P(x, I)=\left(M_{0}\right)$. Let $y \leqq R$ be such that $y(M)=0$ outside $U$ and $y(M)=1$ on $V$, and let $z_{n}$ be chosen in $I(x) \cap \Im\left(M_{0}\right)$ according to Condition (D) so that $z_{n} \rightarrow x$. Let $y_{n}=z_{n} y$, then it can easily be seen that $y_{n}$ belongs locally to $I$ on $S(R)$ and at $\infty$. Hence $x y$ is in $I$ so $x$ belongs locally to $I$ at $M_{0}$. This is a contradiction.

COROLlary 2.6.1 (Generalized Ditkin's Theorem). Let $R$ be a s. s.s. $N^{k}$-algebra satisfying Condition (D), and let $I$ be a closed ideal in $R$. If the boundary of $h(I)$ contains no perfect set then $I=k(h(I))$.

Proof. Pick $y \in k(h(I))$, then $x \in J^{c}(\infty)$ since $R$ is $N^{*}$. Thus, by Condition (D), $x=\lim x_{n}, \quad x_{n} \in[I(x) \cap J(\infty)] \subset \Im^{c}(M)$, all $M \in h(I)$. Thus $P\left(x_{n}, I\right)$ is perfect by Theorem 2. 6. $P\left(x_{n}, I\right) \subset$ boundary of $h(I)$ by Corollary 1.1.1 and the fact that $x_{n}$ belongs locally to $I$ trivially at any point in the interior of $h(I)$. Thus $P\left(x_{n}, I\right)=\phi$ and $x_{n}$ belongs locally to $I$ on $S(R)$ and at $\infty$ (since $x_{n} \in J(\infty)$ ). Thus $x_{n}$ is in $I$ and hence $x$ is in $I$, also.

We conclude this section with examples of the type of theorem one can prove using partitions in cases where property $(P)$ holds in a strengthened sense. These theorems were suggested to the author by Kaplansky's use in [4] of partitions. However, to apply his results we would have to assume continuity of the functions $\|x(M)\|$. Kaplansky is primarily interested in studying $C^{*}$-algebras relative to the structure space of primitive ideals in which case this continuity is equivalent to the structure space being Hausdorff. However, there is no assurance that this is true in general, so we restrict ourselves to the hypothesis that the above mentioned functions are continuous at zero. We will see in $\S 3$ that this much continuity arises in a natural way.

DEFINITION 2.4. Let $R$ be a $G S$-algebra.

(1) For $x \in R$ and $M \in S(R)$ we define $\|x\|_{R}$ to be the norm of the image of $x$ in the $B$-algebra $R / \mathfrak{S}^{c}(M)$, that is, $\|x\|_{M}=$ g.l.b. $\left\{\|y\| \mid y\left(M^{\prime}\right)\right.$ $=x\left(M^{\prime}\right), M^{\prime}$ in some neighborhood of $\left.M\right\}$. We define $\|x\|$ to be 
$\sup \|x\|_{M}, M \in S(R)$. Clearly $\|x\| \leq\|x\|$.

(2) If the norm $\|\cdot\|$ is equivalent to the original norm $\|\cdot\|$ then we say that $R$ is of type $C$.

Definition 2.5. The $B$-algebra $R$ is closed under multiplication by $C(S(R))$ if for $x \in R$ and $f$ a bounded continuous real function on $S(R)$ there exists an element $y \in R$ such that $y(M)=f(M) x(M)$ on $S(R)$. We denote this $y$ by $(f x)$.

The prototype, in a sense, of algebras of type $C$ is the algebra $D_{n}^{\prime}$ in Example 6 of $\S 1$. Here $\|x\| \leqq\|x\| \leq 2\|x\|$. Also, any weakly central $C^{*}$-algebra is of type $C$ and closed under multiplication by $C(S(R))$. Commutative regular $B$-algebras of type $C$ have been studied by Silov in [9] and later papers.

THEOREM 2.7. If $R$ is an $N^{*}$-algebra of type $C$ which is closed under multiplication by $C(S(R))$ then any ideal with compact hull is the kernel of its hull. (In particular, if $R$ has an identity then it is an $N$ algebra.)

Proof. Let $x$ be in $R$ and $F$ be a compact hull with $x \in k(F)$. Then, by Theorem 2.1, for any $M^{\prime} \in F$ and integer $n>0$ there exists $x_{n, M^{\prime}} \in R$ such that $\left\|x_{n, w^{\prime}}\right\|<1 / n$ and $x_{n, w}(M)=x(M)$ for all $M$ in a neighborhood $G_{n}$ of $M^{\prime}$ and all $M$ outside a compact set $K_{n}$. Since $F$ is compact there exist $m$ such open sets $G_{n}\left(M_{i}\right)=G_{i}, i=1, \cdots, m$ covering $F$. Let $K$ be the compact set which accompanies $G_{1}$ as above. Let $\left\{V_{i}\right\}$ cover $F$ with $V_{i} \subset V_{i}^{c} \subset G_{i}, V_{i}^{c}$ compact. If we let $G_{m+1}=\mathscr{C}\left[\cup V_{i}^{c}\right]$ then $G_{1}$, $\cdots, G_{m}, G_{m+1}$ cover $S(R)$ and $G_{m+1}$ is a neighborhood of $\infty$. By a well known lemma $\left[1\right.$, p. 66] there exist $m+1$ continuous real functions $f_{i}$ on $S(R)$ such that $0 \leqq f_{i} \leqq 1, \sum f_{i}=1$ on $S(R)$, and $f_{i}=0$ outside $G_{i}$. Let $x_{n}=\sum_{i=1}^{m}\left(f_{i} x_{n, M_{i}}\right)+\left(f_{m+1} x_{n, M_{1}}\right)$. Clearly $x_{n}(M)=\left[\sum_{i=1}^{m} f_{i}(M)\right] x(M)=x(M)$ on $\cup V_{i}$, a neighborhood of $F$, and also $x_{n}(M)=f_{m+1}(M) x(M)=x(M)$ outside $K \cup \mathscr{C}\left[\bigcup_{i=1}^{n} G_{i}^{c}\right]$, a compact set. It is also clear that $\left\|x_{n}\right\|=\sup \left\|x_{n}\right\|_{k}$ $=\sup \left\|x_{n}(M)\right\|$ since $R$ is $N^{*}$. Finally, $\left\|x_{n}(M)\right\| \leqq \sum f_{i}(M)\left\|x_{n, m_{i}}\right\|$ $+f_{m+1}\left\|x_{n, M_{1}}\right\|<1 / n$ so $\left\|x_{n}\right\|<1 / n$ and, since $R$ is of type $C$, $\left\|x_{n}\right\| \rightarrow 0$. Thus the proof of Theorem 2.1 applies to any ideal with compact hull.

Corollary 2.7.1. If the weakly central algebra $R$ satisfies Condition (2) of Definition 2.4 and has center isomorphic to $C_{0}(\Gamma)$, the algebra of all complex continuous functions vanishing at $\infty$ on the locally compact Hausdorff space $I$, then if $R$ is an $N^{*}$-algebra the conclusion 
of Theorem 2.7 holds.

Proof. Since $C_{0}(\Gamma)$ is a commutative regular $B$-algebra with structure space $\Gamma$, Theorem 1.3 tells us that $R$ is a $G S$-algebra and $\Gamma=$ $S(R)$. Thus the hypotheses of Theorem 2.7 are satisfied.

The condition that $R$ is an $N^{*}$-algebra which appears above can be dropped with an appropriate strengthening of the other conditions.

THEOREM 2.8. Let the GS-algebra $R$ satisfy

(1) $\|\cdot\|$ is equivalent to the norm $\sup \|\cdot(M)\|, M \in S(R)$,

(2) the function $\|x(M)\|$ on $S(R)$ for fixed arbitrary $x \in R$ is continuous at zero, that is, at any point $M \in h(x)$, and at $\infty$, and

(3) $R$ is closed under multiplication by $C(S(R))$.

Then $R$ is Tauberian and the conclusion of Theorem 2.7 holds.

This follows readily from Theorem 2.7 when we observe (following Theorem 3.4) that the above conditions imply that $R$ is a Tauberian $N^{*}$-algebra. Condition (1) implies type $C$.

3. Structure Theorems. In this section we discuss the construction out of primary $B$-algebras of more general $B$-algebras.

Definition 3.1. Let $\Gamma$ be a locally compact Hausdorff space and for each $\alpha \in \Gamma$ let there correspond a primary $B$-algebra $P_{\alpha}$ with norm $\|\cdot\|_{\alpha}$ and unique maximal regular ideal $M_{\alpha}$. The complete direct sum $\sum_{\alpha_{\in} F} P_{\alpha}$ is the totality of functions $x$ defined on $\Gamma$ which satisfy:

$$
\begin{aligned}
& x(\alpha) \in P_{\alpha} \text { for all } \alpha \in \Gamma \text { and } \\
& \|x\|=\sup _{\alpha \in \Gamma}\|x(\alpha)\|_{\alpha}<\infty .
\end{aligned}
$$

A standard elementary proof shows that $\sum P_{\alpha}$ is a $B$-algebra. A closed subalgebra (denoted by $\Sigma^{\prime} P_{\alpha}$ ) of $\sum P_{\alpha}$ is a sub-direct-sum of the $P_{\alpha}$ 's if for each fixed $\alpha$ the set $\left\{x(\alpha) \mid x \in \Sigma^{\prime} P_{\alpha}\right\}$ is all of $P_{\alpha}$.

We list in one definition some additional properties of sub-directsums which will be useful below.

DEFinition 3.2. $\quad \sum^{\prime} P_{\alpha}$ (or, for brevity, $\sum^{\prime}$ ) satisfies Condition (a) if $\left\|x(\alpha)\left(M_{\alpha}\right)\right\|_{\alpha}$ is a continuous function of $\alpha$ on $I^{\top}$ for each fixed 
$x \in \Sigma^{\prime}$,

(b) if $x(\alpha) \in M_{\alpha}$, all $\alpha$, implies that $x=0$,

(c) if $\Sigma^{\prime}$ distinguishes between points of $\Gamma$ in the (weak) sense that $\alpha \neq \beta$ implies that there exists an $x \in \sum^{\prime}$ such that $x(\alpha)\left(M_{\alpha}\right)=0$ and $x(\beta)\left(M_{\beta}\right) \neq 0$,

(d) if $\Sigma^{\prime}$ is closed under multiplication by $C(\Gamma)$, that is, $f x$ is in $\Sigma^{\prime}$ if $f$ is a real bounded continuous function on $\Gamma$ and $x$ is in $\Sigma^{\prime}$,

(e) if $\|x(\alpha)\|_{\alpha}$ is a continuous function of $\alpha$ at any point $\alpha_{0}$ such that $x\left(\alpha_{0}\right)=0$ and at $\infty$ (for any $x \in \Sigma^{\prime}$ we define $x(\infty)$ to be zero).

Definition 3.3. The GS-algebra $R$ is of type $C_{0}$ if $R$ is of type $C$ and the real functions $\|x(M)\|$ are continuous on $S(R)$.

We denote a general element of $S(R)$ by $\alpha$ and the corresponding maximal regular ideal by $M^{\alpha}$. The ideal $\Im\left(M^{\alpha}\right)$ will be denoted by $\Im(\alpha)$.

TheOREM 3.1. Let $R$ be a GS-algebra of type $C$. Then $R=\sum_{\alpha \varepsilon F}^{\prime} P_{\alpha}$ where $\Gamma=S(R)$ and $P_{\alpha}=R / \Im^{c}(\alpha)$ (that is, $R$ is isomorphic and homeomorphic to a sub-direct sum of the primary B-algebras $R / \Im^{c}(\alpha), \alpha \in S(R)$ ) and the sum satisfies Conditions (b), (c), and Condition (e) at finite points. If $R$ is of type $C_{0}$ then the sum satisfies Condition (a). If $R$ is Tauberian then Condition (e) is satisfied at $\infty$.

We omit the proof of this theorem since it is entirely straightforward. It is of interest to note that Condition (e) does not depend upon the GS-condition, but only upon the definition of $\Im(\alpha)$. Also, using the full power of Corollary 1.1.1 we can get a stronger type of separation than that of Condition (c). If $\alpha \neq \beta$ then one can show that there is an $x \in R$ such that $x(\alpha)=0$ and $x(\beta)=1$. However, we shall need only the weaker type of separation.

We turn next to the converse question.

Lemma 3.2.1, Let $\Sigma^{\prime}$ denote $\sum_{\alpha \in \Gamma}^{\prime} P_{\alpha}$ as above. Define $M^{\alpha}$ in $\Sigma^{\prime}$ to be $\left\{x \in \Sigma^{\prime} \mid x(\alpha) \in M_{\alpha}=S\left(P_{\alpha}\right)\right\}$. Then $M^{\alpha}$ is in $S\left(\Sigma^{\prime}\right)$.

This lemma follows immediately from Lemma 1.1.1. In the following we shall always assume that $\Gamma$ is a l.c. Hausdorff space, $P_{\alpha}$ is a primary $B$-algebra with maximal regular ideal $M_{\alpha}$, and $\Sigma^{\prime}$ is a subdirect sum of the $P_{\alpha}$. Unfortunately, we see no way of proving that $\Sigma^{\prime}$ is a $G S$-algebra. Indeed, if no other restrictions are placed on the sum there are examples (commutative) where $S\left(\Sigma^{\prime}\right)$ is not Hausdorff. We can ask, however, whether there is any close tie between $I$ and $S\left(\Sigma^{\prime}\right)$ and in certain cases get a partial answer. 
Theorem 3.2. If $\Sigma^{\prime}$ satisfies Conditions (a) and (c) then $\alpha \rightarrow M^{\alpha}$ is a one-to-one continuous map of $\Gamma^{\prime}$ into $S\left(\Sigma^{\prime}\right)$. If $\Sigma^{\prime}$ satisfies Condition (b) also then the image of $\Gamma$ is dense. If $\Sigma^{\prime}$ is a GS-algebra and satisfies Conditions (a), (b), and (c) then $\Gamma$ is homeomorphic to $S\left(\Sigma^{\prime}\right)$.

Proof. Condition (c) obviously implies that the mapping is one-toone. Let $\Gamma_{s}$ be the image of $\Gamma$ in $S\left(\Sigma^{\prime}\right)$. If $F_{r}$ is in $\Gamma$ and $F_{F} \rightarrow F_{s}$ $=F \cap \Gamma_{s}$ where $F$ is a hull in $S\left(\Sigma^{\prime}\right)$ let $\alpha_{0}$ be in $\Gamma^{\prime}-F_{\Gamma}$. Then $M^{\alpha_{0}}$ is not in $F_{s}$ so it is not in $F$. Thus there exists $x \in \Sigma^{\prime}$ such that $x\left(M^{\alpha}\right)$ $=0, M^{\alpha} \in F_{s}$ and $x\left(M^{\alpha_{0}}\right) \neq 0$. This is equivalent to saying that $x(\alpha)\left(M_{\alpha}\right)$ $=0$ for $\alpha \in F$ and $x\left(\alpha_{0}\right)\left(M_{\alpha_{0}}\right) \neq 0$. Hence, by Condition (a), $\alpha_{0}$ is not in $F_{T}^{c}$ and so $F_{T}$ is closed. Similarly one shows that if the kernel of $F$ is regular then $F_{F}$ is compact, that is, $F_{T}$ is closed in the one-point compactification $\Gamma^{\prime}$ of $\Gamma$. We conclude that $\alpha \rightarrow M^{\alpha}, \infty \rightarrow \infty$ is a one-toone continuous map of $\Gamma^{\prime}$ into $S^{\prime}\left(\Sigma^{\prime}\right)$. Condition (b) insures that $\Gamma_{s}$ is dense in $S\left(\Sigma^{\prime}\right)$ and the rest of the theorem follows from the fact that a one-to-one continuous map of a compact space onto a Hausdorff space is a homeomorphism.

Theorem 3.3. Let $\Sigma^{\prime}$ satisfy Conditions (b) and (d). Then $\Sigma^{\prime}$ is of type $C$. If $\Sigma^{\prime}$ is a GS-algebra and $S\left(\Sigma^{\prime}\right)=\Gamma$ then Condition (d) can be dropped.

Proof. Let $K_{\alpha}$ be the kernel of the natural homomorphism of $\Sigma^{\prime}$ onto $P_{\alpha}$. The map $\alpha \rightarrow M^{\alpha}$ is continuous as we have seen. Let $x \in J(\alpha)$ then $x(M)=0$ for $M$ in a neighborhood of $M^{\alpha}$. This implies that $x(\beta)\left(M_{\beta}\right)=$ 0 for $\beta$ in a neighborhood $N$ of $\alpha$. Let $f \in C(\Gamma)$ be such that $0 \leq f(\beta)$ $\leq 1$ for all $\beta, f(\alpha)=1$ and $f(\beta)=0$ for $\beta$ outside $N$. Then if $y \in \Sigma^{\prime}$ is such that $y(\beta)=f(\beta) x(\beta), \beta \in \Gamma$, it is clear that $y(\beta)\left(M_{\beta}\right)=0$ for all $\beta$. Thus, by (b), $y=0$ so that $x(\alpha)=0$. Hence $J(\alpha) \subset K_{\alpha}$ and, since $K_{\alpha}$ is closed, $J^{c}(\alpha) \subset K_{\alpha}$. Now let $y-x$ be in $\Im^{c}(\alpha)$. Then $(y-x)(\alpha)=0$ so $\|x(\alpha)\|_{\alpha}=\|y(\alpha)\|_{\alpha} \leqq\|y\|$. Thus $\|x(\alpha)\|_{\alpha} \leqq\|x\|_{\mu^{\alpha}}$ (the last norm as in Definition 2.4). Taking the sup over $\Gamma$ we have

$$
\|x\|=\sup \|x(\alpha)\|_{\alpha} \leqq \sup \|x\|_{M^{\alpha}}=\|x\| .
$$

But by definition $\|x\| \leqq\|x\|$ so $\Sigma^{\prime}$ is of type $C$.

In case $\Sigma^{\prime}$ is a $G S$-algebra $\Im^{c}(\alpha)$ has the minimal properties of Theorem 1.2 so, since $K_{\alpha}$ is primary, we can conclude that $\Im^{c}(\alpha) \subset K_{\alpha}$ without assuming Condition (d).

Finally, we consider the question of whether the above mentioned 
decomposition is in any way unique.

Theorem 3.4. Let $\Sigma^{\prime}$ be a GS-algebra and satisfy Conditions (a), (b), (c), (d), and (e). Then $\Gamma^{\prime}=S\left(\Sigma^{\prime}\right), \Sigma^{\prime}$ is Tauberian and of type $C$, and, for each $\alpha, P_{\alpha}$ is equivalent as a B-algebra to $\Sigma^{\prime} / \Im^{c}(\alpha)$.

Proof. Conditions (d) and (e) at $\infty$ clearly imply that $\Sigma^{\prime}$ is Tauberian, so there remains only the last conclusion. This will follow readily if we can prove that the kernel $K_{\alpha}$ of $x \rightarrow x(\alpha)$ equals $\Im^{c}(\alpha)$ for any $\alpha \in \Gamma$. In this event $P_{\alpha}$ and $\Sigma^{\prime} / \mathcal{S}^{\prime}(\alpha)$ are isomorphic and the observation (proof of Theorem 3.3) that $\|x(\alpha)\|_{\alpha} \leq\|x\|_{M^{\alpha}}$ together with the interior mapping theorem show that the isomorphism is an equivalence.

Let $x$ be in $K_{\alpha}$, then $x(\alpha)=0$. Hence by (e) there exists a neighborhood $U_{n}$ of $\alpha$ and a compact set $K_{n}$ such that $\|x(\beta)\|_{\beta}<1 / n$ for $\beta \in U_{n} U$ $\mathscr{C}\left(K_{n}\right)=G_{n}$. If we choose $f \in C\left(I^{\prime}\right)$ such that $0 \leq f(\cdot) \leq 1, f(\beta)=1$ outside $G_{n}$, and $f(\beta)=0$ on $V_{n} \cup \subset\left(F_{n}\right)$, where $V_{n}^{c} \subset U_{n}, F_{n} \supset K_{n}$ and $F_{n}$ is compact, then it is evident that $\left\|y_{n}-x\right\| \leq 2 / n$, and $y_{n}$ is in $\Im(\alpha)$. Thus $x$ is in $\Im^{\prime \prime}(\alpha)$ and $\Im^{c}(\alpha)$ contains $K_{\alpha}$. The opposite inclusion was established in the proof of Theorem 3.3.

We can now complete the proof of Theorem 2.8. The hypotheses of that theorem insure that the sub-direct sum decomposition of $R$ into the simple $B$-algebras $R / M$ which is then possible satisfy all the conditions of Theorem 3.4. The resulting uniqueness conclusion implies that for each $M \in S(R), M=\Im^{c}(M)$, that is, $R$ is an $N^{*}$-algebra.

Finally we observe that the above structure theory has been developed under in one sense the weakest possible condition on the norm of $R$. For if we attempt to weaken the type $C$ assumption by using even smaller ideals than the $\Im^{\prime \prime}(M)$ we lose the fact that the ideals are primary and thus the important connection with the structure space.

\section{REFERENCES}

1. N. Bourbaki, Élements de mathématique, vol. 3, Topologie générale, Actualités Sci. Ind., 1045, Paris, 1948.

2. N. Jacobson, A topology for the set of primitive ideals in an arbitrary ring, Proc. Nat. Acad. Sci. U.S. A., 31 (1945), 333-338.

3. I. Kaplansky, Normed algebras, Duke Math. J., 16 (1949), 399-418.

4. … The structure of certain operator algebras, Trans. Amer. Math. Soc., 70 (1951), 219-255.

5. L.H. Loomis, An introduction to alstract harmonic analysis, New York, 1953. 
6. G.W. Mackey, Commutative Banach algebras, (edited by A. Blair), Harvard University (1952), mimeographed lecture notes.

7. Y. Misonou, On a weakly central operator algebra, Tôhoku Math. J. (2nd series), 4 (1952), 194-202.

8. I. Segal, The group algebra of a locally compact group, Trans. Amer. Math. Soc., 61 (1947), 69-105.

9. G.E. Silov, On regular normed rings, Trav. Inst. Math. Steklov, 21, Moscow, (1947). 10. F.B. Wright, A reduction for algebras of finite type, Ann. of Math., 60 (1954), $560-570$.

Yale University and Amherst College 


\section{PACIFIC JOURNAL OF MATHEMATICS}

\section{EDITORS}

H. L. Royden

Stanford University

Stanford, California

E. HewitT

University of Washington

Seattle 5 , Washington
R. P. Dilworth

California Institute of Technology Pasadena 4, California

E. G. Straus

University of California

Los Angeles 24, California

\section{ASSOCIATE EDITORS}

E. F. BECKENBACH

C. E. BURGESS

H. BUSEMANN

H. FEDERER

\author{
M. HALL \\ P. R. HALMOS \\ V. GANAPATHY IYER \\ R. D. JAMES
}

M. S. KNEBELMAN

I. NIVEN

T. G. OSTROM

M. M. SCHIFFER
J. J. STOKER

G. SZEKERES

F. WOLF

K. YOSIDA

\section{SUPPORTING INSTITUTIONS}

UNIVERSITY OF BRITISH COLUMBIA

CALIFORNIA INSTITUTE OF TECHNOLOGY

UNIVERSITY OF CALIFORNIA

MONTANA STATE UNIVERSITY

UNIVERSITY OF NEVADA

OREGON STATE COLLEGE

UNIVERSITY OF OREGON

UNIVERSITY OF SOUTHERN CALIFORNIA
STANFORD UNIVERSITY

UNIVERSITY OF UTAH

WASHINGTON STATE COLLEGE

UNIVERSITY OF WASHINGTON

AMERICAN MATHEMATICAL SOCIETY CALIFORNIA RESEARCH CORPORATION HUGHES AIRCRAFT COMPANY 


\section{Pacific Journal of Mathematics}

\section{Vol. 6, No. $1 \quad$ November, 1956}

David Blackwell, An analog of the minimax theorem for vector payoffs..... 1

L. W. Cohen, A non-archimedian measure in the space of real

sequences ..................................... 9

George Bernard Dantzig, Constructive proof of the Min-Max theorem ..... 25

Jim Douglas, On the numerical integration of quasilinear parabolic

differential equations ............................... 35

James Michael Gardner Fell, A note on abstract measure ............. 43

Isidore Isaac Hirschman, Jr., A note on orthogonal systems . . . . . . . . . . 47

Frank Harary, On the number of dissimilar line-subgraphs of a given

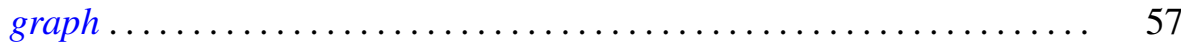

Newton Seymour Hawley, Complex bundles with Abelian group......... 65

Alan Jerome Hoffman, Morris Newman, Ernst Gabor Straus and Olga

Taussky, On the number of absolute points of a correlation ...........

Ernst Gabor Straus and Olga Taussky, Remark on the preceding paper.

Algebraic equations satisfied by roots of natural numbers . . ........ 97

Ralph D. James, Summable trigonometric series ................. 99

Gerald R. Mac Lane, Limits of rational functions . . . . . . . . . . . . . . . 111

F. Oberhettinger, Note on the Lerch zeta function ................. 117

Gerald C. Preston, On locally compact totally disconnected Abelian groups and their character groups ........................... 121

Vikramaditya Singh and W. J. Thron, On the number of singular points, located on the unit circle, of certain functions represented by

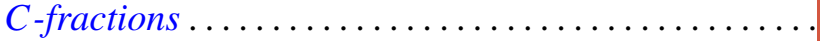

Sherman K. Stein, The symmetry function in a convex body ... 145 Edwin Weiss, Boundedness in topological rings.............

Albert Leon Whiteman, A sum connected with the series for the partition

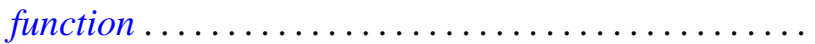

Alfred B. Willcox, Some structure theorems for a class of Banach algebras.

Joseph Lawrence Zemmer, Some remarks on p-rings and their Boolean geometry... 